\title{
CPCGI confers neuroprotection by enhancing blood circulation and neurological function in cerebral ischemia/reperfusion rats
}

\author{
YIFENG MIAO, RAN WANG, HUI WU, SHAOFENG YANG and YONGMING QIU \\ Department of Neurosurgery, Renji Hospital, South Campus, \\ Shanghai Jiaotong University School of Medicine, Shanghai 201112, P.R. China
}

Received August 31, 2018; Accepted May 9, 2019

DOI: $10.3892 / \mathrm{mmr} .2019 .10472$

\begin{abstract}
The current study used a rat middle cerebral artery occlusion (MCAO) model with the aim to explore the effects of compound porcine cerebroside and ganglioside injection (CPCGI) on brain ischemia/reperfusion injury in rats. Improvement in the infarct-side microcirculation and the overall recovery of neurological function were detected by triphenyltetrazolium chloride staining, laser speckle blood flow monitoring, latex perfusion, immunofluorescence and immunoblotting. The results revealed that administration of CPCGI for 7 consecutive days following ischemic stroke contributed to the recovery of neurological function and the reduction of cerebral infarct volume in rats. Blood flow monitoring results demonstrated that the administration of CPCGI effectively promoted cerebral blood flow following stroke, and contributed to the protection of the ischemic side blood vessels. In addition, CPCGI treatment increased the numbers of new blood vessels in the peripheral ischemic region, and upregulated the expression levels of vascular endothelial growth factor, angiopoietin 1 and its receptor TEK receptor tyrosine kinase, fibroblast growth factor and Wnt signaling pathway-associated proteins. Taken together, the present results indicated that CPCGI improved the blood circulation and neurological function following cerebral ischemia/reperfusion in rats.
\end{abstract}

\section{Introduction}

Ischemic stroke is a type of acute cerebrovascular disease that is usually caused by cerebral thrombosis and results in irreversible effects, including microvascular damage, neuronal death and destruction of the blood-brain barrier, in a short period of time (1-5). At present, rapid thrombolytic therapy is commonly used in the treatment of the disease (6). Compound

Correspondence to: Dr Yongming Qiu, Department of Neurosurgery, Renji Hospital, South Campus, Shanghai Jiaotong University School of Medicine, 2000 Jiangyue Road, Shanghai 201112, P.R. China E-mail: yongmingqiu25@163.com

Key words: infarction, middle cerebral artery, cerebrosides, gangliosides, ischemia, reperfusion, stroke, neurology tripeptide injection is used clinically to treat acute and chronic cerebrovascular diseases, including cerebral thrombosis, cerebral embolism and cerebral palsy, as well as brain dysfunction caused by brain trauma and cerebrovascular diseases (brain insufficiency, cerebral infarction) (7-9). Compound porcine cerebroside and ganglioside injection (CPCGI: Drug approval no. H22026472; Buchang Pharmaceutical Group Ltd., Jilin, China), which was approved by the China Food and Drug Administration in 2010, is used to treat arteriosclerosis, thrombophlebitis, capillary hemorrhage and edema caused by increased vascular permeability $(10,11)$.

The cerebral microcirculation network refers to the blood vessel system with a diameter of $<200 \mu \mathrm{m}$ in the brain, consisting of arterioles, capillaries and venules, which can regulate cerebral blood flow, transport nutrients to the neurons and glial cells and remove waste products (12-14). The blood circulation of the large blood vessels is powered by the heart, whereas microcirculation depends upon small arteries, arterioles and capillaries to perform the function of contraction and relaxation (15).

Following the occurrence of ischemic stroke, cerebral microcirculation is blocked, accompanied by changes in multiple brain structures and functions (3). It primarily affects the function of the blood-brain barrier (16). Due to cerebral ischemia and hypoxia, the intimate connection between cerebral vascular endothelial cells is destroyed, the microvascular basement membrane is damaged and the permeability of the vascular wall is increased, resulting in punctiform hemorrhage and brain edema $(17,18)$. Additionally, the vasomotion of cerebral arterioles is inhibited and the blood cannot reach the capillaries controlled by the arterioles, leading to the injury and necrosis of the neurons $(19,20)$. Moreover, the structure of small blood vessels is damaged, which provokes cerebral arterial stenosis or occlusion and a decrease in collateral circulation, thereby further aggravating the disturbance in microcirculation (21).

Previous studies have demonstrated that the improvement of cerebral microcirculation by drug administration may increase the blood flow of the infarcted side, which is beneficial for the repair, regeneration and recovery of nerve functions (22-25). The current study aimed to explore the effects of CPCGI injection on brain ischemia/reperfusion injury in the focal transient middle cerebral artery occlusion (MCAO) rat models. 


\section{Materials and methods}

Ethics statement. The current study was approved by the Ethics Committee of Renji Hospital, South Campus, Shanghai Jiaotong University School of Medicine (Shanghai, China).

Establishment of rat models of permanent MCAO. A total of 24 male Sprague-Dawley rats (Shanghai Laboratory Animal Center, Shanghai, China) aged 7-8 weeks and weighing 250-300 g were housed under standard laboratory conditions at a temperature of $20-22^{\circ} \mathrm{C}$ and 12 -h light/dark cycles and were provided with food and water ad libitum. Rats were anesthetized by intraperitoneal injection of $7 \%$ chloral hydrate ( $350 \mathrm{mg} / \mathrm{kg}$; Bax \& Company, Cambridge, UK) and then placed in a supine position. After disinfection, the skin was cut open along the midline of the neck and the muscle layers were separated by blunt dissection. The right common carotid artery, the external carotid artery and the internal carotid artery were carefully separated, and the external carotid artery and the common carotid artery were ligated. The pterygopalatine artery was isolated along the internal carotid artery. The distal ends of the pterygopalatine and internal carotid arteries were temporarily clamped with a rat artery clip. A small incision was made at the bifurcation between the external and internal carotid arteries using a pair of ophthalmic scissors. A nylon suture with a spherical-shaped head $(0.265 \mathrm{~mm}$ in diameter with a mark made $18 \mathrm{~mm}$ from the head) was inserted into the incision. The time that resistance was encountered was recorded as the ischemia time, and the suture was removed when resistance was no longer encountered the length of the inserted nylon suture was $18-20 \mathrm{~mm}$. The wound was sutured after ligating the incision at the bifurcation between the external and internal carotid arteries.

Study design. The rats were randomly divided into the sham operation group (rats were anesthetized without the insertion of nylon suture), MCAO rat model group, low dose and high dose groups ( 0.83 and $2.5 \mathrm{ml} / \mathrm{kg} / \mathrm{day}$ CPCGI, respectively; Jilin Buchang Pharmaceutical Group Co., Ltd.) for 7 consecutive days. The intraperitoneal injection was delivered immediately after the model was established. Sham and model groups received an equivalent volume of saline. All the animals were sacrificed after 7 days of modeling.

Monitoring cerebral blood flow changes in cortex by laser speckle. The PeriCam PSI system (Thermo Fisher Scientific, Inc., Waltham, MA, USA) is a blood perfusion imaging system based on the laser speckle contrast analysis technology. The instrument excites a laser with a certain frequency. Different degrees of reflected waves are received after the laser beam is irradiated to the tissues. Blood with different flow velocities induces different reflected waves. Therefore, different color images are produced according to the variations of blood flow volume and these can be used to monitor the blood flow velocity and blood flow volume in real-time. This technology has been widely applied to monitor the blood flow changes following cerebral stroke (18). In the current study, the PeriCam PSI system was adopted to monitor the changes of blood flow in the right cerebral cortex before and 7 days after the establishment of the MCAO model. After anesthetizing the rats, the fur over the top of the skull was shaved, a $3 \mathrm{~cm}$ incision was created along the sagittal suture, and the skin was pulled apart with the sutures. A dental drill was used to evenly thin the skull until the blood vessels were clearly visible. At this time, the PeriCam PSI system was used to monitor the blood flow volume and this measurement was used as the baseline level of the blood flow volume of the cerebral cortex. After the MCAO model was established, the blood flow at the same location was repeatedly monitored. After treatment for 7 days, the blood flow in each group was repeatedly monitored. The duration of each monitoring was $20 \mathrm{~min}$. The image processing was performed by the PimSoft data analysis software version 13.0 (Beyotime Institute of Biotechnology, Haimen, China). The ischemic region was manually delineated to statistically analyze the blood flow volume. The rats were kept in the same position during the measurements to facilitate data processing.

TTC staining. Following reperfusion, rat brains were removed and frozen at $-80^{\circ} \mathrm{C}$ for $5 \mathrm{~min}$. Sections $(2 \mathrm{~mm})$ were made using a rodent brain matrix (HEAD Biotechnology Co., Ltd., Beijing, China) and stained for $20 \mathrm{~min}$ at $37^{\circ} \mathrm{C}$ with $2 \%$ 2,3,5-triphenyltetrazolium chloride monohydrate (TTC; Sigma-Aldrich; Merck KGaA, Darmstadt, Germany) and were then fixed with $4 \%$ formaldehyde. The infarct volume was calculated as previously described (26). The sections were scanned and the infarct area was calculated using ImageJ analysis software version 1.46 (National Institutes of Health, Bethesda, MD, USA).

Western blot analysis. Protein was extracted from brain tissues with radioimmunoprecipitation assay lysis buffer (Santa Cruz Biotechnology, Inc., Dallas, TX, USA) with protease inhibitors and phosphatase inhibitors. Proteins were determined by BCA Protein Assay kit (Pierce; Thermo Fisher Scientific, Inc.); $50 \mu \mathrm{g}$ protein was separated via SDS-PAGE on a $12 \%$ gel. The separated proteins were subsequently transferred onto polyvinylidene fluoride membrane. The membrane was blocked with 5\% non-fat milk at room temperature for $1.5 \mathrm{~h}$. The membrane was incubated with rabbit polyclonal primary antibodies purchased from Santa Cruz Biotechnology, Inc. at $4^{\circ} \mathrm{C}$ overnight. Primary antibodies against vascular endothelial growth factor (cat. no. 03265, VEGF; 1:1,000), angiopoietin 1 (cat. no. 21783, ANGPT1; 1:1,000), TEK receptor tyrosine kinase (cat. no. 25309, TEK; 1:1,000), fibroblast growth factor (cat. no. 37021, FGF; 1:500), frizzled (cat. no. 22301, Fzd; 1:500), $\beta$-catenin (cat. no. 70225, 1:800), Wnt family member 7A (cat. no. 13762, WNT7A; 1:500) and $\beta$-actin (cat. no. 24971, 1:800) were used. The membrane was subsequently incubated with horseradish peroxidase-conjugated goat anti-rabbit secondary antibody (cat. no. 2041; 1:1,000; Santa Cruz Biotechnology, Inc.) for $2 \mathrm{~h}$ at room temperature. ECL chromogenic substrates were used for quantitative measurement using Quantity One software (version 4.6; Bio-Rad Laboratories, Inc., Hercules, CA, USA).

Neurological function assessment. Neurological function was graded on a scale of 0-12 (normal score, 0 ; maximal score, 12) as previously described by Belayev et al (27). Tests were conducted by an observer blinded to the treatment groups. The rats were lifted by the tail and suspended in the air ( 2 points): 
0 points, no obvious neurological function deficit; 1 point, slight flexion of the contralateral limb of the infarcted limb; 2 points, significant flexion of the contralateral limb of the infarcted limb (28).

Limb placement was assessed by the visual sub-test (anterior, lateral) and tactile sub-test (anterior, lateral; 8 points): 0 points, normal limb placement; 1 point, reaction delay time $\leq 2 \mathrm{sec} ; 2$ points, reaction delayed time $>2 \mathrm{sec}$. Proprioceptive sub-test ( 2 points): 0 points, equal strength to the contralateral limb; 1 point, slightly stronger than the contralateral limb; 2 points, weaker than the contralateral limb (29).

Cerebral perfusion and brain extraction in rats. Following the MCAO procedure, rats were anesthetized with an intraperitoneal injection of $7 \%$ chloral hydrate $(350 \mathrm{mg} / \mathrm{kg}$; Sigma-Aldrich; Merck KGaA). No animals exhibited signs of peritonitis following the administration of chloral hydrate. The rats were placed on the operating table in a supine position and the abdominal muscle layer was cut open, and the ribs and diaphragm were transected to fully expose the heart. Cardiac puncture was performed in the apex of the left ventricle with a $10 \mathrm{ml}$ syringe filled with $0.9 \%$ physiological saline (Sigma-Aldrich, Merck KGaA). A small incision was created at the right atrial appendage using a pair of ophthalmic scissors. The rat liver became white and the heartbeat slowed down. A portion of $200 \mathrm{ml}$ of $2 \%$ paraformaldehyde perfusion was performed at a fast and then slow speed. The signs of successful perfusion were as follows: At the beginning of perfusion, the rats were twitched violently and the tails tilted upwards. After the perfusion, the hind limbs of the rats were stretched straight, the tail was erected and the limbs were pale and rigid.

The muscles of the back of the neck were removed and the brain tissues were collected with curved forceps to maintain the integrity of the brain. The brain tissues were placed in the prepared $2 \%$ paraformaldehyde solution for $10 \mathrm{~min}$ and stored at $4^{\circ} \mathrm{C}$. Initially, the brain tissues floated on the surface, while one week later they sank to the bottom of the solution. The fixation solution was replaced every $\sim 48 \mathrm{~h}$, and subsequently the brain tissues were prepared for frozen sections.

Latex perfusion procedures. The rats were anesthetized and placed on a plate in a supine position. A syringe filled with $0.9 \%$ physiological saline was used to puncture the apex of the left ventricle and a small incision was created at the right atrial appendage with a pair of ophthalmic scissors. A total of $50 \mathrm{ml}$ of $0.9 \%$ physiological saline was gently injected. The rat liver became white and the heartbeat was slowed down. Approximately $20 \mathrm{ml}$ of warm latex perfusion fluid (Sigma-Aldrich; Merck KGaA) was injected into the heart at a speed of $10 \mathrm{ml} / \mathrm{min}$. The signs of perfusion were as follows: The forelimbs of the rats were slightly gray. When the skull was cut open, the cerebral vessels were filled with black latex perfusion fluid, whereas the brain tissues remained white. After perfusion, the rat whole brain was carefully collected and immediately observed under an inverted microscope at x10 magnification (Nikon Ti; Nikon Corporation, Tokyo, Japan).

Immunofluorescence assay. Brains were fixed by perfusion with $4 \%$ paraformaldehyde for $3 \mathrm{~h}$ at $4^{\circ} \mathrm{C}$. Brains were embedded in paraffin and sections (thickness, $20 \mu \mathrm{m}$ ) were blocked $4^{\circ} \mathrm{C}$ for $1.5 \mathrm{~h}$ by $1.5 \% \mathrm{BSA}$ in $0.01 \mathrm{M} \mathrm{PBS}$, the sections were incubated with primary antibodies overnight at $4^{\circ} \mathrm{C}$. The antibodies used were as follows: Anti-lectin (cat. no. ab64693; 1:200; Abcam), anti-Ki67 (cat. no. ab833; 1:800; Abcam), anti-VE-cadherin (cat. no. ab231227; 1:200; Abcam) or anti-phosphorylated-focal adhesion kinase (p-FAK; cat. no. ab4792; 1:500; Abcam). Sections were then washed with $0.01 \mathrm{M}$ PBS three times, and incubated with horseradish peroxidase-conjugated goat anti-rabbit secondary antibody (cat. no. 2041; 1:1,000; Santa Cruz Biotechnology, Inc.) for $1 \mathrm{~h}$ at room temperature. All sections were washed three times with $0.01 \mathrm{M}$ PBS for $10 \mathrm{~min}$ in the dark and mounted on a coverslip with mounting medium containing DAPI at room temperature for $2 \mathrm{~h}$ (Santa Cruz Biotechnology, Inc.).

The slides were visualized using a fluorescence microscope (Olympus Corporation; magnification, x20) and analyzed by Image-Pro Plus software version 7.0 (Media Cybernetics, Inc., Rockville, MD, USA).

Statistical analysis. Statistical analysis was performed using SPSS software (version 18; SPSS, Inc., Chicago, IL, USA). Data were presented as mean \pm SD of at least three independent experiments. One-way analysis of variance and post hoc test were used to determine the differences between multiple groups. $\mathrm{P}<0.05$ was considered to indicate a statistically significant difference.

\section{Results}

Protective effects of CPCGI on the brain of rats with ischemic stroke. At 7 days following the establishment of MCAO, the Belayev score (27) was significantly different between the model and sham operation groups $(\mathrm{P}<0.001$; Fig. 1A). Compared with the model group, the Belayev scores in the LD and HD groups were significantly decreased $(\mathrm{P}<0.01$ and $\mathrm{P}<0.001$, respectively; Fig. 1A), suggesting that CPCGI effectively reduced the neurological impairment and contributed to rehabilitation following ischemic stroke. The infarction volume of the rat brains at 7 days after model establishment was evaluated by TTC staining. The infarction volume (white part) in the brains of the CPCGI-treated groups was smaller compared with the model group (Fig. 1B). The percentages of the infarction volume in the whole brain were $23.10 \%$ in the model group, $15.57 \%$ in the LD group and $11.57 \%$ in the HD group. Compared with the model group, there was a significant decrease in the cerebral infarction volume in the LD $(\mathrm{P}<0.01)$ and HD groups $(\mathrm{P}<0.001$; Fig. 1C).

\section{Effects of CPCGI administration on cerebral vessels and blood} flow in rats with cerebral stroke. The PeriCam PSI system was used to monitor the blood flow volume before and after model establishment and at 7 days postoperatively. The changes in blood flow values revealed that at the same ischemic degree, the cerebral blood flow of the infarcted side was improved in the LD group compared with the model group at 7 days following CPCGI administration ( $\mathrm{P}<0.05$; Fig. $2 \mathrm{~A}$ and $\mathrm{B})$. The blood flow in the HD group was also significantly improved compared with the model group $(\mathrm{P}<0.01$; Fig. 2A and $\mathrm{B})$. The quantity and morphology of the vessels in the infarcted 
A

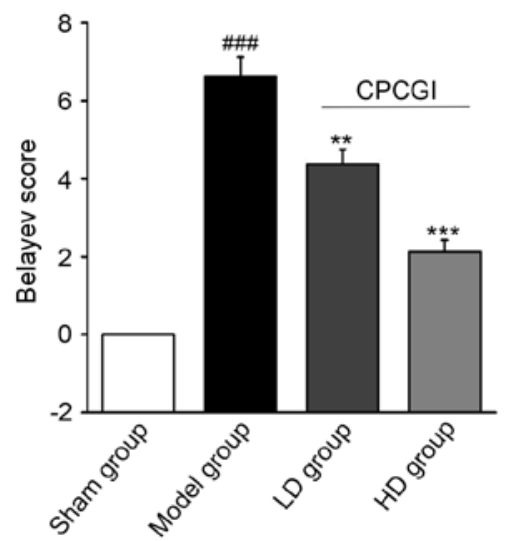

B

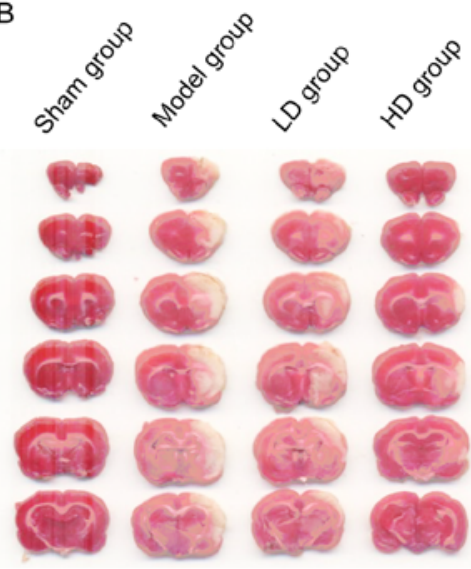

C

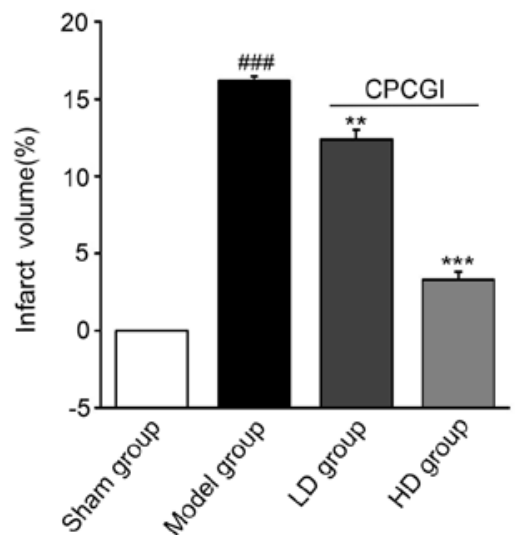

Figure 1. Protective effects of CPCGI on the brain of rats with ischemic stroke. (A) Belayev score in the model and sham operation groups. (B) Cerebral infarction volume in the CPCGI dosage groups by triphenyltetrazolium chloride staining. (C) Quantitative analysis of cerebral infarction volume. ${ }^{\# \# ~} \mathrm{P}<0.001$ vs. sham operation group; ${ }^{* *} \mathrm{P}<0.01$ and ${ }^{* * * *} \mathrm{P}<0.001$ vs. model group. CPCGI, compound porcine cerebroside and ganglioside injection; LD, low dose; HD, high dose.

A

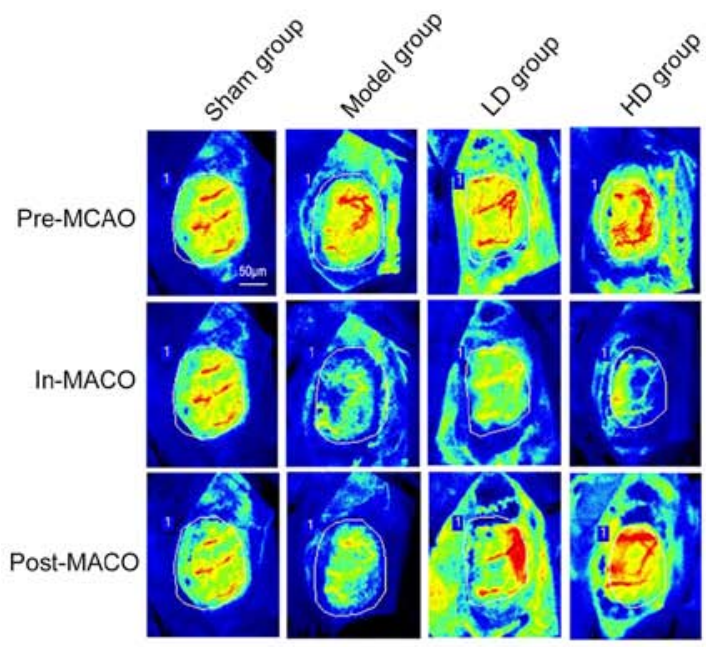

C

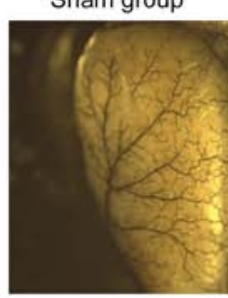

B
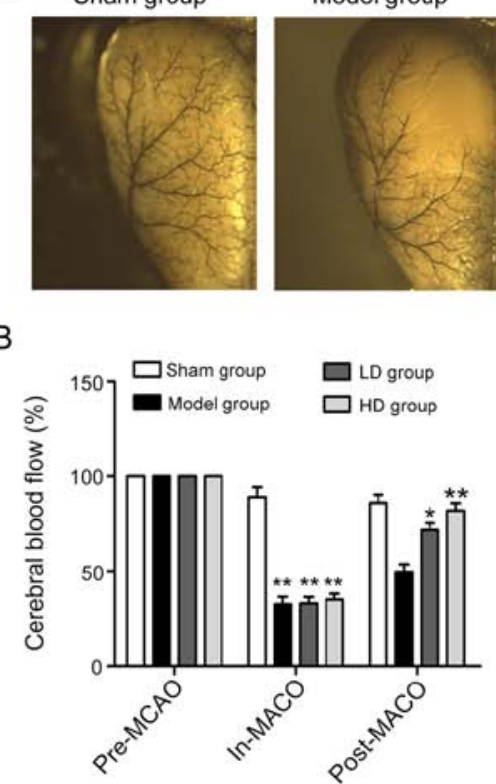

LD group

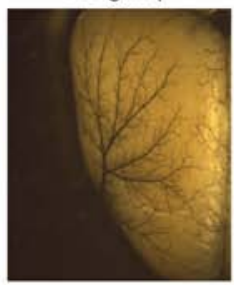

D

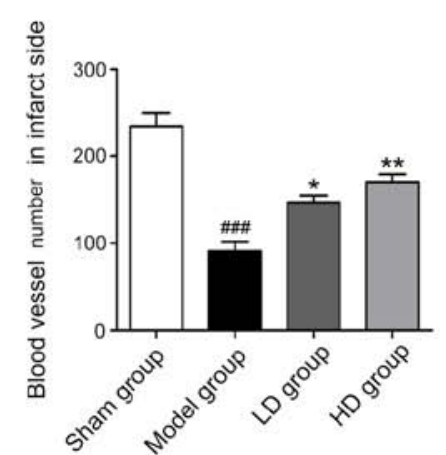

Figure 2. Effects of CPGCI on cerebral vessels and blood flow in rats with cerebral stroke. (A) Blood flow volume before, during and after MCAO model establishment and 7 days postoperatively. Injection region was outlined. Yellow and red areas indicate high blood perfusion. Blue and green areas indicate low blood perfusion. (B) Quantitative analysis of the blood flow volume. (C) The number of vessels in the parietal cortex 7 days after model establishment. (D) Quantitative analysis of the number of vessels in the parietal cortex 7 days after model establishment. ${ }^{\# \# \#} \mathrm{P}<0.01$ vs. sham operation group; ${ }^{*} \mathrm{P}<0.05$ and ${ }^{* *} \mathrm{P}<0.01$ vs. model group. MCAO, middle cerebral artery occlusion; LD, low dose; HD, high dose; In-MCAO, during MACO.

cerebral parietal cortex in each group were then detected by latex perfusion. The number of vessels in the model, LD and HD groups was significantly decreased compared with the sham operation group (Fig. $2 \mathrm{C}$ and D). The number of vessels in the LD and HD groups was significantly increased compared with the model group (Fig. 2C and D), indicating that CPCGI was beneficial for the preservation of the cerebral cortex vessels following stroke.

Next, the expression levels of vascular endothelial (VE)-cadherin in microvessels adjacent to the ischemic cerebral infarction were examined by lectin $^{+} / \mathrm{VE}$-cadherin ${ }^{+}$ immunofluorescent staining. The expression of VE-cadherin in the CPCGI-treated groups was markedly upregulated compared with the model group (Fig. 3A). Quantification of the lectin ${ }^{+} / \mathrm{VE}$-cadherin ${ }^{+}$double-positive staining revealed that VE-cadherin expression in microvessels was significantly increased in the HD group compared with the model group $(\mathrm{P}<0.01$; Fig. 3A and $\mathrm{B})$. These results suggested that CPCGI may maintain the integrity of blood vessels by upregulating the expression of VE-cadherin in the microvessels surrounding the infarction region, preserving the blood supply of small blood vessels and contributing to the protection and reconstruction of blood-brain barrier.

Effects of CPCGI administration on cerebral angiogenesis following stroke. Immunofluorescent staining revealed that the 
A

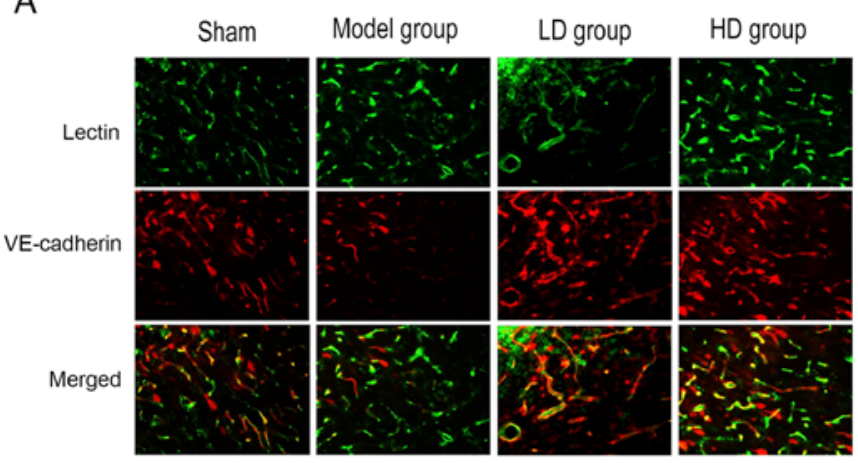

B

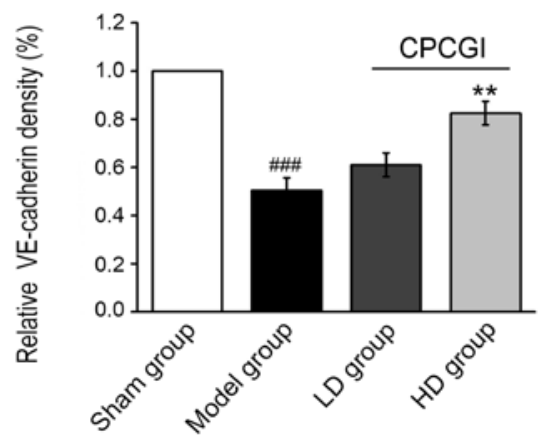

Figure 3. Expression levels of VE-cadherin in microvessels adjacent to the ischemic cerebral infarction. (A) Representative images of VE-cadherin and lectin immunofluorescent staining in the CPCGI groups and the model group (magnification, $x 20)$. (B) Quantitative analysis of VE-cadherin expression in the different groups. ${ }^{\# \#} \mathrm{P}<0.001$ vs. sham operation group; ${ }^{* *} \mathrm{P}<0.01$ vs. model group. VE, vascular endothelial; CPCGI, compound porcine cerebroside and ganglioside injection; LD, low dose; HD, high dose.

number of Ki67-labeled new vessels at 7 days postoperatively in the HD group was significantly higher compared with the model group $(\mathrm{P}<0.05$; Fig. $4 \mathrm{~A}$ and $\mathrm{B})$. The pseudopodia of the sprouting vessels were labeled by $\mathrm{lectin}^{+} / \mathrm{p}-\mathrm{FAK}^{+}$immunofluorescent dual staining. p-FAK was highly expressed in the axonal structure of the microvessels compared with the blood vessels. The expression levels of p-FAK in the peripheral cortex of the infarcted region in the LD and HD groups were significantly upregulated compared with the model group $(\mathrm{P}<0.05$ and $\mathrm{P}<0.001$, respectively; Fig. 4C and $\mathrm{D})$.

Effects of CPCGI administration on VEGF, ANGPT1, TEK, FGF and Wnt signaling proteins following stroke. The relative protein expression levels of VEGF, ANG1, TEK and FGF were detected by western blot analysis in each group at 7 days following the stroke. In the model group, the expression levels of VEGF, ANGPT1, TEK and FGF were significantly upregulated compared with the sham operation group (Fig. 5A). The expression levels of each protein in the LD and HD groups were significantly increased compared with the model group $(\mathrm{P}<0.05$ and $\mathrm{P}<0.001$, respectively; Fig. 5A). In addition, following CPCGI treatment for 7 days, the expression levels of WNT7A, Fzd and $\beta$-catenin were slightly upregulated, however the differences were not statistically significant (Fig. 5B).

\section{Discussion}

As a major challenge in geriatric medicine worldwide, cerebral stroke is characterized by high morbidity, recurrence and mortality rates, as well as severe irreversible injury which is difficult to treat (30). Although the treatment and rehabilitation of patients with stroke is actively researched, rapid thrombolysis remains the mainstay of treatment following stroke $(31,32)$.

In the current study, the neurological function of rats with ischemia-reperfusion following 7 days of intraperitoneal injection of CPCGI was evaluated. Compared with the model group, the administration of CPCGI for 7 consecutive days gradually restored function in rats with ischemic cerebral injury. TTC staining demonstrated that the volume of cerebral infarction in the CPCGI group was significantly decreased compared with the control group, suggesting that CPCGI may ameliorate cerebral infarction and contribute to post-stroke recovery. In the present investigation, laser speckle was adopted to monitor the variations of the cerebral cortex parietal blood flow before and after model establishment, and at 7 days following CPCGI administration. CPCGI improved the blood flow of the infarcted side in rats with stroke. Additionally, a higher dose of CPCGI exerted more significant effects compared with a lower dose, suggesting that administration of CPCGI following stroke contributes to the maintenance of blood flow. However, whether this phenomenon results from small vascular protection or angiogenesis remains to be investigated.

The latex perfusion results in the current study revealed that the number of blood vessels in the ischemic cerebral hemisphere cortex was significantly increased compared with the model group following 7 days of CPCGI injection, indicating that CPCGI has a protective effect on the blood vessels of the infarcted side in rats with stroke. Lectin ${ }^{+} /$VE-cadherin ${ }^{+}$ immunofluorescent dual staining revealed that the expression levels of VE-cadherin in the microvessels of rats with cerebral ischemia were significantly upregulated compared with the model group, suggesting that CPCGI may maintain the vascular integrity in the infarcted area and the blood supply of microvessels and may contribute to the protection and reconstruction of the blood-brain barrier.

Multiple studies have demonstrated that angiogenesis adjacent to the infarcted area serves a significant role in the recovery of neurological function in patients with stroke (33-35). A large quantity of new microvessels gradually forms a new vascular network through growth, adhesion and fusion. The new vascular system is formed after the maturation of the new microvessels and their network reconstruction (36). The newly-formed vascular system gradually substitutes the damaged microvascular system, supplying blood to the infarcted-penumbral brain tissues to provide the nutrients required to promote neural repair (37). In the current study, $\mathrm{Ki}^{+} / \mathrm{lectin}^{+}$immunofluorescent staining demonstrated that administration of CPCGI for 7 days may effectively promote angiogenesis and contribute to enhancing the blood supply surrounding the infarcted area.

Taken together, intraperitoneal injection of CPCGI accelerated the recovery of neurological function and reduced the volume of cerebral infarction in rats with ischemic stroke, indicating that CPCGI may be conducive to the recovery of neurological function and cerebral microcirculation following stroke. Furthermore, CPCGI exerted significant effects on angiogenesis, and this was associated with increased expression of VEGF, ANGPT1, TEK, FGF and Wnt signaling proteins. WNT7A, ANGPT1 and TEK may regulate a variety of cellular 

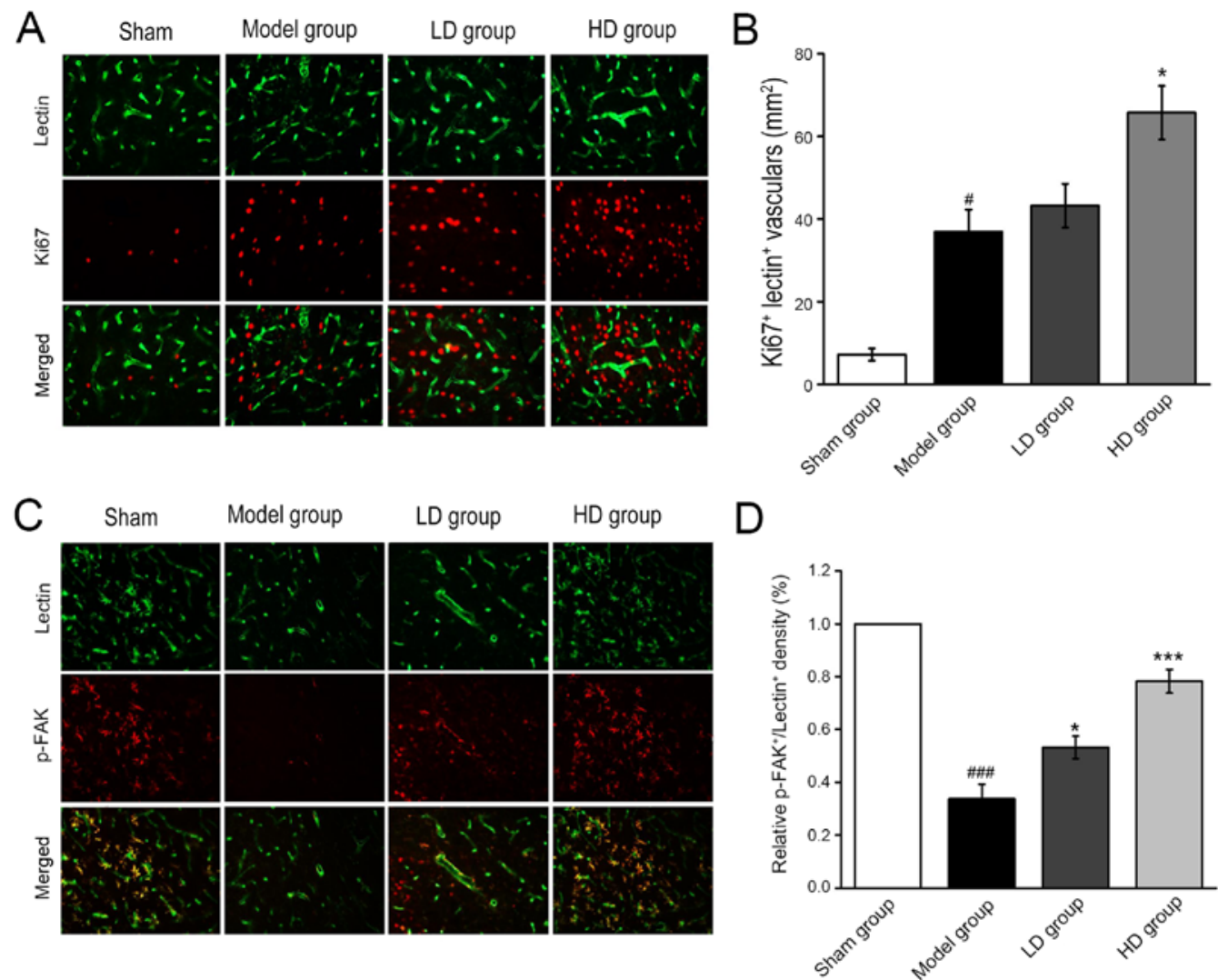

Figure 4. Immunofluorescent staining reveals Ki67- and p-FAK-positive newly formed vessels after 7 days of CPCGI administration. (A) Representative images (magnification, $\mathrm{x} 20$ ) and (B) quantification of newly sprouting vessels, labeled by ki67 $7^{+} /$lectin $^{+}$immunofluorescent dual staining (magnification, $\mathrm{x} 20$ ) (C) Representative images and (D) quantification of pseudopodia of the sprouting vessels in the peripheral cortex of the infarcted region, by $\mathrm{p}-\mathrm{FAK}^{+} / \mathrm{lectin}^{+}$ immunofluorescent dual staining. ${ }^{~} \mathrm{P}<0.05,{ }^{\# \# "} \mathrm{P}<0.001$ vs. sham operation group; $\mathrm{P}<0.05,{ }^{* * *} \mathrm{P}<0.001$ vs. model group. CPCGI, compound porcine cerebroside and ganglioside injection; p-FAK, phosphorylated focal adhesion kinase; LD, low dose; HD, high dose.
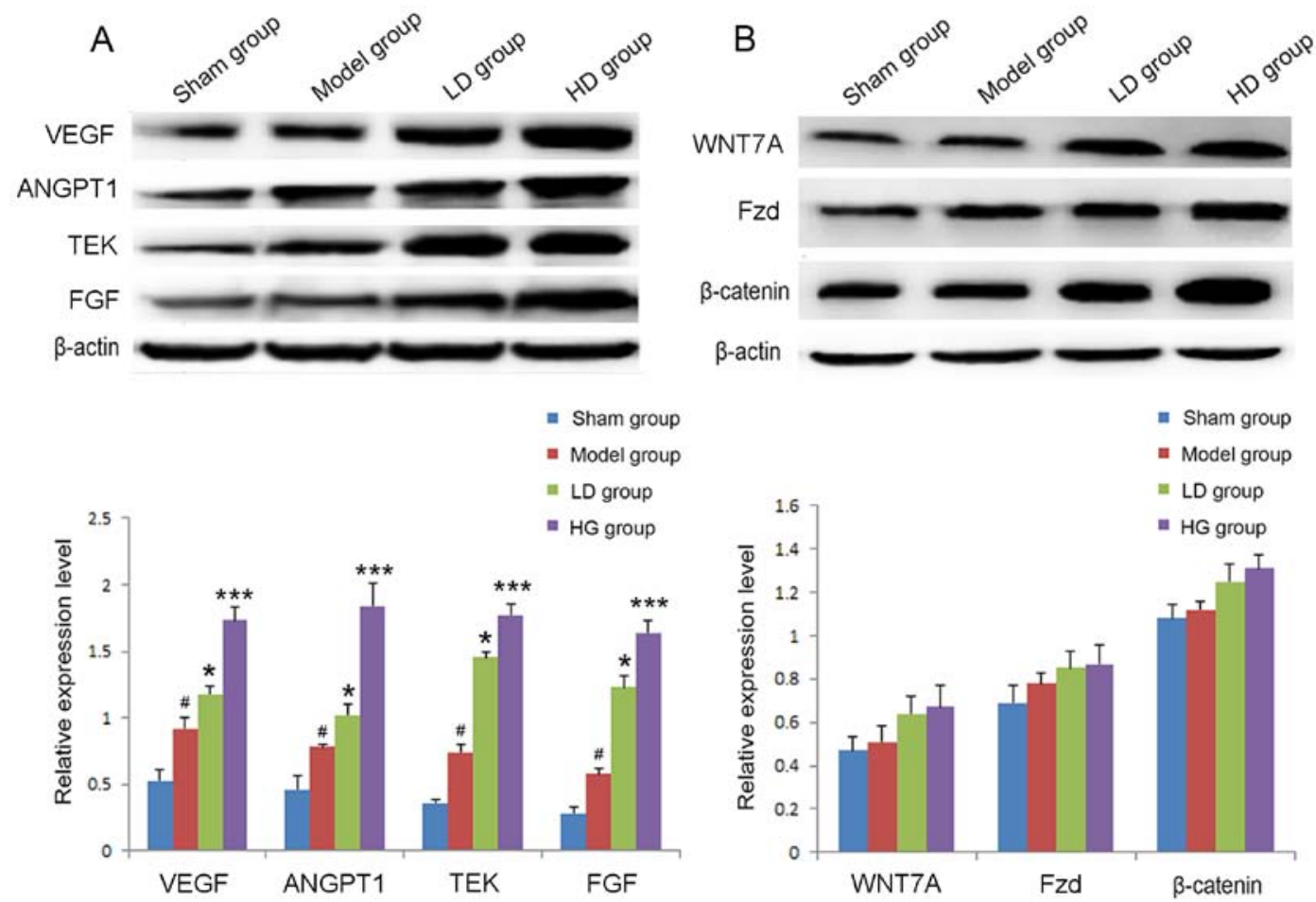

Figure 5. Relative expression levels of VEGF, ANGPT1, TEK and FGF 7 days after stroke in each group. (A) The expression levels of VEGF, ANGPT1, TEK and FGF were detected by western blot analysis. (B) The expression levels of WNT7A, Fzd and $\beta$-catenin were detected by western blot analysis. ${ }^{*} \mathrm{P}<0.05$ vs. sham operation group; ${ }^{*} \mathrm{P}<0.05,{ }^{* * *} \mathrm{P}<0.001$ vs. model group. VEGF, vascular endothelial growth factor; ANGPT1, angiopoietin 1 ; TEK, TEK receptor tyrosine kinase; FGF, fibroblast growth factor; WNT7A Wnt family member 7A; Fzd, frizzled. 
and developmental pathways, and increase cell proliferation via activation of the canonical WNT/ $\beta$-catenin pathway $(38,39)$.

In conclusion, the results obtained in the current study demonstrated that CPCGI exerted positive effects on cerebral microcirculation following ischemic stroke, suggesting that it may be a promising therapeutic option for patients with cerebral stroke in clinical practice.

\section{Acknowledgements}

Not applicable.

\section{Funding}

No funding was received.

\section{Availability of data and materials}

All data generated or analyzed during this study are included in this published article.

\section{Authors' contributions}

YQ conceived the study. YM and SY designed and performed the experiments. RW participated in the experiments and drafted the manuscript. HW performed statistical analysis and interpreted the data. All authors read and approved the final manuscript.

\section{Ethics approval and consent to participate}

The current study was approved by the Ethics Committee of Renji Hospital, South Campus, Shanghai Jiaotong University School of Medicine (Shanghai, China).

\section{Patient consent for publication}

Not applicable.

\section{Competing interests}

The authors declare that they have no competing interests.

\section{References}

1. Chikuda H: Response: RE: Ischemic stroke after cervical spine injury: Analysis of 11,005 patients using the Japanese Diagnosis Procedure Combination database. Spine J 15: 2593-2594, 2015.

2. Arauz A, Romano JG, Ruiz-Franco A, Shang T, Dong C, Rundek T, Koch S, Hernández-Curiel B, Pacheco J, Rojas P, et al: Differences in lipid profiles in two Hispanic ischemic stroke populations. Int J Stroke 9: 394-399, 2014.

3. Behrouz R: The risk of ischemic stroke in major rheumatic disorders. J Neuroimmunol 277: 1-5, 2014.

4. Liu NN, Dong ZL and Han LL: MicroRNA-410 inhibition of the TIMP2-dependent MAPK pathway confers neuroprotection against oxidative stress-induced apoptosis after ischemic stroke in mice. Brain Res Bull 143: 45-57, 2018.

5. Tuo QZ, Liuyang ZY, Lei P, Yan X, Shentu YP, Liang JW, Zhou H, Pei L, Xiong Y, Hou TY, et al: Zinc induces CDK5 activation and neuronal death through CDK5-Tyr15 phosphorylation in ischemic stroke. Cell Death Dis 9: 870, 2018.

6. Ní Chróinín D, Asplund K, Åsberg S, Callaly E, CuadradoGodia E, Díez-Tejedor E, Di Napoli M, Engelter ST, Furie KL, Giannopoulos S, et al: Statin therapy and outcome after ischemic stroke: Systematic review and meta-analysis of observational studies and randomized trials. Stroke 44: 448-456, 2013.
7. Dmitrieva VG, Povarova OV, Skvortsova VI, Limborska SA, Myasoedov NF and Dergunova LV: Semax and Pro-Gly-Pro activate the transcription of neurotrophins and their receptor genes after cerebral ischemia. Cell Mol Neurobiol 30: 71-79, 2010.

8. Terent A and Ronquist G: Cerebrospinal fluid markers of disturbed brain cell metabolism in patients with stroke and global cerebral ischemia. Acta Neurol Scand 62: 327-335, 1980.

9. Wang M, Zhang Y, Feng L, Zheng J, Fan S, Liu J, Yang N, Liu Y and Zuo P: Compound porcine cerebroside and ganglioside injection attenuates cerebral ischemia-reperfusion injury in rats by targeting multiple cellular processes. Neuropsychiatr Dis Treat 13: 927-935, 2017.

10. Doronin II, Vishnyakova PA, Kholodenko IV, Ponomarev ED, Ryazantsev DY, Molotkovskaya IM and Kholodenko RV: Ganglioside GD2 in reception and transduction of cell death signal in tumor cells. BMC Cancer 14: 295, 2014.

11. Novak A, Režić Mužinić N, Cikeš Čulić V, Božić J, Tičinović Kurir T, Ferhatović L, Puljak L and Markotić A: Renal distribution of ganglioside GM3 in rat models of types 1 and 2 diabetes. J Physiol Biochem 69: 727-735, 2013.

12. Dostal P, Schreiberova J, Dostalova V, Dostalova V Jr, Tyll T, Paral J, Abdo I, Cihlo M, Astapenko D and Turek Z: Effects of hypertonic saline and mannitol on cortical cerebral microcirculation in a rabbit craniotomy model. BMC Anesthesiol 15: 88, 2015.

13. Morikawa T, Kajimura M, Nakamura T, Hishiki T, Nakanishi T, Yukutake Y, Nagahata Y, Ishikawa M, Hattori K, Takenouchi T, et al: Hypoxic regulation of the cerebral microcirculation is mediated by a carbon monoxide-sensitive hydrogen sulfide pathway. Proc Natl Acad Sci USA 109: 1293-1298, 2012.

14. Chen Y, Li Q, Tang J, Feng H and Zhang JH: The evolving roles of pericyte in early brain injury after subarachnoid hemorrhage. Brain Res 1623: 110-122, 2015.

15. Bragin DE, Bush RC and Nemoto EM: Effect of cerebral perfusion pressure on cerebral cortical microvascular shunting at high intracranial pressure in rats. Stroke 44: 177-181, 2013.

16. Engelhardt B and Liebner S: Novel insights into the development and maintenance of the blood-brain barrier. Cell Tissue Res 355: 687-699, 2014.

17. Zhao H, Wang J, Gao L, Wang R, Liu X, Gao Z, Tao Z, Xu C, Song J, Ji X and Luo Y: MiRNA-424 protects against permanent focal cerebral ischemia injury in mice involving suppressing microglia activation. Stroke 44: 1706-1713, 2013.

18. Zhang L, Niu W, He Z, Zhang Q, Wu Y, Jiang C, Tang C, Hu Y and Jia J: Autophagy suppression by exercise pretreatment and p38 inhibition is neuroprotective in cerebral ischemia. Brain Res 1587: 127-132, 2014.

19. Hao HF, Liu LM, Liu YY, Liu J, Yan L, Pan CS, Wang MX, Wang CS, Fan JY, Gao YS and Han JY: Inhibitory effect of rhynchophylline on contraction of cerebral arterioles to endothelin 1: Role of rho kinase. J Ethnopharmacol 155: 147-153, 2014.

20. Lapi D, Scuri R and Colantuoni A: Trigeminal cardiac reflex and cerebral blood flow regulation. Front Neurosci 10: 470, 2016.

21. Chen H, Wu B, Zhu G, Wintermark M, Wu X, Su Z, Xu X, Tian C, Ma L, Zhang W and Lou X: Permeability imaging as a biomarker of leptomeningeal collateral flow in patients with intracranial arterial stenosis. Cell Biochem Biophys 71: 1273-1279, 2015.

22. Gong P, Zhao S, Wang J, Yang Z, Qian J, Wu X, Cahoon J and Tang W: Mild hypothermia preserves cerebral cortex microcirculation after resuscitation in a rat model of cardiac arrest. Resuscitation 97: 109-114, 2015.

23. Cabrales P, Zanini GM, Meays D, Frangos JA and Carvalho LJ: Nitric oxide protection against murine cerebral malaria is associated with improved cerebral microcirculatory physiology. J Infect Dis 203: 1454-1463, 2011.

24. Kim KJ and Filosa JA: Advanced in vitro approach to study neurovascular coupling mechanisms in the brain microcirculation. J Physiol 590: 1757-1770, 2012.

25. Wu Q, Qi L, Li H, Mao L, Yang M, Xie R, Yang X, Wang J, Zhang Z, Kong J and Sun B: Roflumilast reduces cerebral inflammation in a rat model of experimental subarachnoid hemorrhage. Inflammation 40: 1245-1253, 2017.

26. Chen HJ, Shen YC, Shiao YJ, Liou KT, Hsu WH, Hsieh PH, Lee CY, Chen YR and Lin YL: Multiplex brain proteomic analysis revealed the molecular therapeutic effects of Buyang Huanwu decoction on cerebral ischemic stroke mice. PLoS One 10: e0140823, 2015.

27. Belayev L, Alonso OF, Busto R, Zhao W and Ginsberg MD: Middle cerebral artery occlusion in the rat by intraluminal suture. Neurological and pathological evaluation of an improved model. Stroke 27: 1616-1623, 1996. 
28. Bederson JB, Pitts LH, Tsuji M, Nishimura MC, Davis RL and Bartkowski H: Rat middle cerebral artery occlusion: Evaluation of the model and development of a neurologic examination. Stroke 17: 472-476, 1986

29. De Ryck M, Van Reempts J, Borgers M, Wauquier A and Janssen PA: Photochemical stroke model: Flunarizine prevents sensorimotor deficits after neocortical infarcts in rats. Stroke 20: 1383-1390, 1989

30. Zhang JB, Jü XH, Wang J, Sun HR and Li F: Serum cystatin C and cerebral microbleeds in patients with acute cerebral stroke. J Clin Neurosci 21: 268-273, 2014.

31. Sakr SA, El-Rasheedy WA, Ramadan MM, El-Menshawy I, Mahfouz E and Bayoumi M: Association between left atrial appendage morphology evaluated by trans-esophageal echocardiography and ischemic cerebral stroke in patients with atrial fibrillation. Int Heart J 56: 329-334, 2015.

32. Liutkiene G, Stropus R, Dabuzinskiene A and Pilmane M: Structural changes of the human superior cervical ganglion following ischemic stroke. Medicina (Kaunas) 43: 390-398, 2007.

33. Chin Y, Kishi M, Sekino M, Nakajo F, Abe Y, Terazono Y, Hiroyuki O, Kato F, Koizumi S, Gachet $\mathrm{C}$ and Hisatsune T: Involvement of glial P2Y(1) receptors in cognitive deficit after focal cerebral stroke in a rodent model. J Neuroinflammation 10: 95, 2013.
34. Pavlidis E, Spagnoli C, Duca M, Ormitti F, Magnani C and Pisani F: Neonatal forearm compartment syndrome: Look for cerebral stroke. J Pediatr 164: 427.e1, 2014.

35. Tamiya S, Yoshida Y, Harada S, Nakamoto K and Tokuyama S: Establishment of a central post-stroke pain model using global cerebral ischaemic mice. J Pharm Pharmacol 65: 615-620, 2013.

36. Yu Q, Chu M, Wang H, Lu S, Gao H, Li P, Gan Y, Shi H, Liang W, Chen J and Gao Y: Sevoflurane preconditioning protects blood-brain-barrier against brain ischemia. Front Biosci (Elite Ed) 3: 978-988, 2011

37. Lapi D, Vagnani S, Sapio D, Mastantuono T, Sabatino L, Paterni $\mathrm{M}$ and Colantuoni A: Long-term remodeling of rat pial microcirculation after transient middle cerebral artery occlusion and reperfusion. J Vasc Res 50: 332-345, 2013.

38. Pazhohan A, Amidi F, Akbari-Asbagh F, Seyedrezazadeh E, Farzadi L, Khodarahmin M, Mehdinejadiani S and Sobhani A: The Wnt $/ \beta$-catenin signaling in endometriosis, the expression of total and active forms of $\beta$-catenin, total and inactive forms of glycogen synthase kinase- $3 \beta$, WNT7a and DICKKOPF-1. Eur J Obstet Gynecol Reprod Biol 220: 1-5, 2018.

39. Katoh $\mathrm{Y}$ and Katoh M: Comparative integromics on Angiopoietin family members. Int J Mol Med 17: 1145-1149, 2006. 